\title{
The Structure of the SIrP-hTrx1 Complex Sheds Light on the Autoinhibition Mechanism of the Type-III Secretion System Effectors of the NEL Family
}

\author{
Samira Zouhir*, Joaquín Bernal-Bayard ${ }^{\dagger}$, Mar Cordero-Alba ${ }^{\dagger}$, Elena Cardenal-Muñoz ${ }^{\dagger}$ Beatriz \\ Guimaraes $^{\ddagger}$, Noureddine Lazar ${ }^{\S}$, Francisco Ramos-Morales ${ }^{\dagger} \&$ Sylvie Nessler $^{\star \S}$ \\ * Laboratoire d'Enzymologie et Biochimie Structurales (LEBS), CNRS UPR 3082, 91198 Gif sur Yvette, \\ France \\ $\dagger$ Departamento de Genética, Facultad de Biología, Universidad de Sevilla, Apartado 1095,41080 Sevilla, \\ Spain \\ \$ Synchrotron SOLEIL, 91192 Gif sur Yvette, France \\ $\S$ Institut de Biochimie et Biophysique Moléculaire et Cellulaire (IBBMC) University Paris-Sud 11, UMR \\ 8619,91405 Orsay, France
}

Corresponding authors: Sylvie Nessler, Institut de Biochimie et Biophysique Moléculaire et Cellulaire (IBBMC) University Paris-Sud 11, UMR 8619, 91405 Orsay, France, Tel.: (+33)169157966; Fax: (+33)169853715; E-mail: sylvie.nessler@u-psud.fr; Francisco Ramos-Morales, Departamento de Genética, Facultad de Biología, Universidad de Sevilla, Avda. Reina Mercedes 6, 41012 Sevilla, Spain, Tel.: (+34)954557107; Fax: (+34)954557104; E-mail: framos@us.es

SZ and JBB contributed equally to this work

Present addresses:

SZ: Laboratório Nacional de Biociências (LNBio), CNPEM, Campinas, Brazil

JBB: Département de Microbiologie, Institut Pasteur, 75724 Paris cedex 15, France

Short title: Structural analysis of the SlrP/Trx1 complex

Keywords: Salmonella infection; Effector-host protein interaction; Crystal structure; LRR domain; Ubiquitination; Novel bacterial E3 ligase 


\begin{abstract}
Salmonella infections are a leading cause of bacterial foodborne illness in the United States and the European Union. Antimicrobial therapy is often administered to treat the infection but increasing isolates are being detected that demonstrate resistance to multiple antibiotics. Salmonella enterica contains two virulence related type-III secretion systems (T3SS): one promotes invasion of the intestine and the other one mediates systemic disease. Both of them secrete the SlrP protein acting as E3 ubiquitin ligase in human host cells where it targets thioredoxin-1 (Trx 1). SlrP belongs to the NEL family of bacterial E3 ubiquitin ligases that have been observed in two distinct autoinhibitory conformations. We solved the 3D structure of the SlrP/Trx 1 complex and determined the Trx 1 ubiquitination site. The description of the substrate-binding mode sheds light on the first step of the activation mechanism of SlrP. Comparison with the available structural data of other NEL effectors allowed us to gain new insights into their autoinhibitory mechanism. We propose a molecular mechanism for the regulation of SlrP in which structural constraints sequestrating the NEL domain would be sequentially released. This work thus constitutes a new milestone in the understanding of how these T3SS effectors influence pathogen virulence. It also provides the fundamental basis for future development of new antimicrobials.
\end{abstract}




\section{INTRODUCTION}

Infectious diseases are a major concern of human health and the steady rise of antibiotic resistance urges for new strategies to reduce bacterial infections. While traditional antibiotics kill or slow bacterial growth, an important emerging strategy to combat pathogens seeks to block the ability of bacteria to harm the host by inhibiting bacterial virulence factors. Many pathogenic bacteria are able to manipulate the cellular integrity of host cells by secreting proteins in the extracellular environment or inside host cells. In Gram-negative pathogens, the translocated proteins, named effectors, are directly delivered into the hostcell cytoplasm through the type-III secretion system (T3SS), which displays a needle-like structure [1]. The T3SS offers an interest as target for the design of new antibiotics [2]. However, another therapeutic approach consists in targeting the secreted effectors that, once delivered by the T3SS, play critical roles in manipulating the host cell to allow for bacterial invasion, intracellular survival and proliferation $[3,4]$. The nature of the effectors is very variable $[5,6]$ and they utilize three main strategies to modify host cell function [7]: they directly bind to, covalently modify, or mimic endogenous host cell proteins.

Although ubiquitin (Ub) is absent from bacteria, some species secrete effectors displaying E3 ubiquitin ligase activity, which are able to subvert the ubiquitination pathway of the human host cell $[8,9]$. Ubiquitination consists in the covalent attachment of one or more ubiquitin molecules to lysine residues of substrate proteins. This post-translational modification constitutes an attractive target for a wide range of pathogens because it regulates many pathways in eukaryotic cells such as protein degradation by the $26 \mathrm{~S}$ proteasome, DNA repair or activation of protein kinases [10]. Ubiquitination involves a cascade of three enzymes [11]. First, the ubiquitin-activating enzyme (E1) activates the ubiquitin molecule through an ATP-dependent reaction to create a thioester bond between the E1 catalytic cysteine and the carboxylate of the ubiquitin C-terminal glycine. In the second step, the activated ubiquitin is transferred by a transthiolation reaction from $\mathrm{E} 1$ to a member of the ubiquitin-conjugating enzyme (E2) family. Finally, in the presence of a ubiquitin-protein ligase (E3), which binds to specific substrates, ubiquitin is conjugated to the target protein by the formation of an isopeptide bond between the carboxyl terminus of ubiquitin and the $\varepsilon$-amino group of a lysine residue of the target protein [11]. E3 ligases determine the substrate specificity of ubiquitination and have been classified into two main classes, the HECT and RING finger families [12]. The more prevalent RING-type E3 ligases bring together an E2-Ub conjugate and a substrate protein, and in most cases, ubiquitin is transferred directly from the $\mathrm{E} 2$ to the substrate. By contrast, HECT-type E3 ligases are directly involved in the catalytic process: ubiquitin is transferred from the E2 enzyme to the catalytic cysteine of the HECT domain, and then to a lysine residue of the target [13]. Most known bacterial E3 ligases do not share sequence similarity with eukaryotic E3 ligases [14]. They display however structural similarity with the RING-type [15] or HECT-type enzymes [16].

Recently, a new family of bacterial E3 ligases has been discovered among T3SS effectors of a subset of pathogenic bacteria [17]. They are characterized by the presence of a N-terminal leucine-rich repeat (LRR) domain involved in protein/protein interactions [18] and a C-terminal catalytic domain displaying an $\alpha$-helical fold completely distinct from either the RING or HECT domains [19]. This catalytic domain was named NEL for "Novel E3 Ligase". The presence of the N-terminal substrate-binding domain and the requirement for a catalytic cysteine are reminiscent of the mechanism of HECT-type E3-ligases [20]. Similarly, the catalytic cysteine located within a conserved CXD motif of the NEL domain acts as a nucleophile to catalyze ubiquitin transfer through a transthiolation reaction.

Comparison of the structures of the full-length effectors IpaH3 from Shigella flexneri (PDB ID 3CVR) [21] and SspH2 from Salmonella enterica serovar Typhimurium (PDB ID 3G06) [22] revealed that the LRR and NEL domains, which are linked by a flexible loop, display drastic distinct relative orientations. $\mathrm{SspH} 2$ is in a closed autoinhibitory conformation burying the catalytic cysteine in the domain interface. The NEL domain is orientated toward the concave face of the banana shaped LRR domain. A bridging interaction is observed between the catalytic and $\alpha \mathrm{G}-\alpha \mathrm{H}$ loops of the NEL domain and the N-terminus of the linker region. In the IpaH3 structure the $\alpha \mathrm{G}-\alpha \mathrm{H}$ loop interacts with the convex side of the LRR domain while the catalytic loop is exposed to the solvent. This open conformation was first interpreted as the active state of the LRR-NEL effectors [14]. However, a recent mutational analysis [23] demonstrated 
that both conformations protect the LRR-NEL effectors from autoubiquitination and it was proposed that these two autoinhibition modes could be released upon substrate binding [23].

In a previous study [24] we showed that one of the members of this LRR-NEL family of T3SS effectors, the Salmonella secreted protein SlrP, specifically recognizes the reduced form of human thioredoxin-1 (Trx1). Ubiquitination of this essential redox protein resulted in an increased susceptibility of the host cells to death. In the present work, we solved the crystal structure of the SlrP/Trx1 complex and identified the Trx 1 ubiquitination site. The comparison of these new results with the available data concerning other LRR-NEL proteins finally allowed us to assess the role of substrate binding in the autoinhibition mechanism of these T3SS effectors.

\section{EXPERIMENTAL}

\section{Bacterial and yeast strains and culture}

Bacterial strains used in this study are Escherichia coli BL21(DE3) (Stratagene), M15 (Qiagen), DH5 $\alpha$ [25] and XL1-Blue [26], and Salmonella enterica serovar Typhimurium 14028. Culture medium for bacteria was Luria-Bertani (LB) broth. Antibiotics were used at the following final concentrations: ampicillin, $100 \mu \mathrm{g} / \mathrm{ml}$; kanamycin, $50 \mu \mathrm{g} / \mathrm{ml}$; tetracycline, $20 \mu \mathrm{g} / \mathrm{ml}$. Saccharomyces cerevisiae strains used are Y2HGold (Clontech) and Y187 [27]. Culture media for yeast were YPD and yeast drop out lacking the appropriate supplements: tryptophan to select for the presence of pGBKT7 derivatives, leucine for pGADT7 derivatives, histidine and adenine to check interactions. Solid media contained agar at $1.5 \%$ final concentration.

\section{Plasmids and mutagenesis}

To obtain the plasmid to generate 6 His recombinant proteins, DNA from strain 14028 was used as a template for PCR amplification. The amplified fragments were digested with BamHI and SalI and ligated with BamHI/SalI-digested pQE30 (Qiagen). Plasmid pREP4 (Qiagen), that constitutively expresses the lac repressor protein encoded by the lacI gene, was used to regulate expression of 6 His recombinant proteins. Derivatives of pGEX-4T-1 and pGEX-4T-3 (GE Healthcare) were used for production of GST fusion proteins. To generate point mutations in $\operatorname{slr} P$ and $\operatorname{trx} 1$, the previously described plasmids [24] pIZ1635 (pGBKT7-SlrP), pIZ1710 (pGADT7-Trx1) and pIZ1712 (pGEX-4T-1-Trx1) were used as templates. Amplification reactions were carried out in a T100 thermal cycler (BioRad) using KAPA HiFi DNA polymerase (Kapa Biosystems) and the primers listed in Table S1. The PCR cycling parameters were set up as following: (i) initial denaturation, $3 \mathrm{~min}$ at $95^{\circ} \mathrm{C}$; (ii) 17 cycles of denaturation $\left(98^{\circ} \mathrm{C}, 20 \mathrm{~s}\right.$ ), annealing $\left(62^{\circ} \mathrm{C}, 15 \mathrm{~s}\right)$, and extension $\left(72{ }^{\circ} \mathrm{C}, 30 \mathrm{~s} / \mathrm{kb}\right)$; (iii) final extension $\left(72^{\circ} \mathrm{C}, 5 \mathrm{~min}\right)$. Products were digested with $1 \mu \mathrm{l}$ of DpnI $(10 \mathrm{U} / \mu \mathrm{l})$ for $1 \mathrm{~h}$ at $37^{\circ} \mathrm{C}$ and $E$. coli DH5 $\alpha$ was transformed with these digested plasmids. All PCR constructs were confirmed by DNA sequencing using an automated DNA sequencer (Stab Vida, Oeiras, Portugal).

\section{Yeast two-hybrid methods}

Plasmids were introduced into Saccharomyces cerevisiae strains Y2HGold or Y187 using the lithium acetate transformation procedure, as previously described [28]. Yeast transformed with the appropriate plasmids were mixed onto YPD plates and incubated at $30^{\circ} \mathrm{C}$ overnight. The mating mixtures were plated in yeast drop out medium lacking tryptophan and leucine. Plates were incubated at $30^{\circ} \mathrm{C}$ for 3 days and then colonies were patched on the same medium and replica-plated on medium lacking tryptophan, leucine, histidine and adenine, to check for the expression of the HIS 3 and ADE2 reporters.

\section{Protein preparation}

The human trxl gene and the slrP gene fragments from Salmonella enterica serovar Typhimurium corresponding to residues 1 to 140 and 140 to 765 were cloned into pQE30 plasmids and expressed with an N-terminal 6His-tag as recombinant proteins in E. coli M15/pREP4 strain. The oligonucleotides used 
for cloning are shown in Table S1. The same production and purification protocols were used for all proteins, except that $5 \mathrm{mM} \beta$-mercapto-ethanol was added in all Trx buffers to stabilize the reduced form of the protein that has been shown to interact with SlrP [24]. Bacteria were grown in LB medium containing $100 \mu \mathrm{g} / \mathrm{ml}$ of ampicillin and $50 \mu \mathrm{g} / \mathrm{ml}$ of kanamycin at $37^{\circ} \mathrm{C}$. At mid-exponential growth phase $\left(\mathrm{OD}_{600}=0.6-0.8\right)$, the temperature was decreased to $30^{\circ} \mathrm{C}$ and $1 \mathrm{mM}$ of IPTG was added to induce expression and incubation was pursued for $4 \mathrm{~h}$. The cells were harvested by centrifugation at $5000 \mathrm{rpm}$ for $20 \mathrm{~min}$ at $4^{\circ} \mathrm{C}$. The cell pellet was resuspended in lysis buffer $(20 \mathrm{mM}$ Tris- $\mathrm{HCl}, \mathrm{pH} 7.5,300 \mathrm{mM} \mathrm{NaCl}$ and $10 \mathrm{mM}$ imidazole, $+/-5 \mathrm{mM} \beta$-mercapto-ethanol). A commercial mixture of protease inhibitors (Sigma) was added to the suspension and lysis was performed by sonication. The cell lysate was centrifuged at $15000 \mathrm{~g}$ for $30 \mathrm{~min}$ at $4^{\circ} \mathrm{C}$ and the supernatant filtered $(0.20 \mu \mathrm{m})$ before loading onto a $\mathrm{Ni}^{2+}$-NTA column equilibrated with lysis buffer. After extensive washing the 6 His-tagged protein was eluted with $300 \mathrm{mM}$ imidazole in lysis buffer. The eluted fraction was filtered $(0.20 \mu \mathrm{m})$ and injected onto a size exclusion column (Superdex S200 26/60 for S1rP and Superdex S75 26/60 for Trx1) equilibrated in $20 \mathrm{mM}$ Tris-HCl, $\mathrm{pH} 7.5,150 \mathrm{mM} \mathrm{NaCl}, 5 \mathrm{mM} \beta$-mercapto-ethanol. The purification was performed at $5^{\circ} \mathrm{C}$ on an HPLC ÄKTA Purifier (GE Pharmacia). The purified proteins were concentrated by ultrafiltration (Vivaspin $30 \mathrm{kDa}$ and $5 \mathrm{kDa}$ for SlrP and Trx1, respectively) and stored aliquoted at $20^{\circ} \mathrm{C}$.

Selenomethionine-labeled SlrP was produced from E. coli strain M15/pREP4/pIZ1784 grown overnight at $28^{\circ} \mathrm{C}$ in the presence of $100 \mu \mathrm{g} / \mathrm{ml}$ of ampicilin and $25 \mu \mathrm{g} / \mathrm{ml}$ of kanamicin in M9 minimal medium supplemented with $100 \mu \mathrm{g} / \mathrm{ml}$ of Lys, Thr and Phe, and $50 \mu \mathrm{g} / \mathrm{ml}$ of Leu, Ile, Val and Met. At mid-exponential growth phase $\left(\mathrm{OD}_{600}=0.6\right)$, the cells were harvested by centrifugation at $5000 \mathrm{rpm}$ for 10 min at room temperature and resuspended in fresh M9 mediun supplemented as before except for methionine that was replaced by $50 \mu \mathrm{g} / \mathrm{ml}$ of selenomethionine. After incubating 30 min at $37^{\circ} \mathrm{C}$, expression was induced with $1 \mathrm{mM}$ of IPTG for $4 \mathrm{~h}$ at $37^{\circ} \mathrm{C}$. The purification protocol was unchanged.

Expression of the GST fusion proteins was induced by the addition of $1 \mathrm{mM}$ isopropyl $\beta-D-$ thiogalactoside to E. coli BL21(DE3) bacteria containing pGEX-4T-1, pGEX-4T-3 or their derivatives, and the fusion proteins were isolated from bacterial lysates by affinity chromatography with glutathioneagarose beads (Sigma). For lysis, bacteria were sonicated in Nonidet P-40 buffer (10 mM Tris-HCl, pH 7.4, $150 \mathrm{mM} \mathrm{NaCl}, 10 \%$ glycerol, $1 \%$ Nonidet P-40, $1 \%$ aprotinin, $1 \mathrm{mM}$ phenylmethylsulfonyl fluoride, $1 \mu \mathrm{g} / \mathrm{ml}$ pepstatin, and $1 \mu \mathrm{g} / \mathrm{ml}$ leupeptin).

\section{Size-exclusion chromatography - Multi-angle laser light scattering measurements}

The chromatographic analyses of SlrP and Trx proteins were performed using the SEC-TDA equipment by Viscotek (Malvern, France). Protein samples of $100 \mu 1$ were loaded at a flow rate of $0.5 \mathrm{ml} / \mathrm{min}$ onto a Agilent Bio Sec-3 column and eluted in $20 \mathrm{mM}$ Tris pH 7.5, $150 \mathrm{mM} \mathrm{NaCl}, 5 \mathrm{mM} \beta$-mercapto-ethanol. Elution was followed by a UV-Visible spectrophotometer, a differential refractometer, a $7^{\circ}$ Low Angle Light Scattering detector, a $90^{\circ}$ Right Angle Light Scattering detector and a differential pressure viscometer. The instrument was calibrated using bovine serum (Sigma-Aldrich). The OmniSEC software program was used for the acquisition and analysis of the data.

\section{Pull-down experiments}

Bacterial extracts from M15/pREP4 strains expressing 6 His proteins were incubated at $4^{\circ} \mathrm{C}$ for $3 \mathrm{~h}$ with GST or GST-Trx proteins bound to glutathione-coupled agarose beads. The precipitates were washed six times in Nonidet P-40 buffer followed by SDS-PAGE. The gel was blotted onto a nitrocellulose membrane and probed with monoclonal anti-His antibody (1:3000; GE Healthcare). Goat anti-mouse horseradish peroxidase-conjugated antibodies (BioRad) were used as secondary antibodies. Detection was via chemiluminescence procedures (Pierce).

\section{Crystal structure determination}

Co-crystallization conditions were determined at $18^{\circ} \mathrm{C}$ with various concentrations and ratio of purified SlrP and Trx1 proteins. Commercial crystallization kits were screened using a nanodrop 
crystallization Cartesian robot (Genomics Solutions, UK). Co-crystals were obtained in both Qiagen kit solutions MB Class II ${ }^{\circ} 63\left(0.1 \mathrm{M} \mathrm{MgCl}_{2}, 0.1 \mathrm{M} \mathrm{NaCl}, 0.1 \mathrm{M}\right.$ HEPES, pH7.5, $12 \%$ PEG 4000) and PEG-II $\mathrm{n}^{\circ} 94(0.2 \mathrm{M} \mathrm{Mg}$ Acetate, 10\% PEG 8000). The co-crystals were manually reproduced and optimized at $18^{\circ} \mathrm{C}$ using the hanging drop method in a crystallization solution containing $0.1 \mathrm{M} \mathrm{MgCl}_{2}, 0.2 \mathrm{M} \mathrm{NaCl}$, $0.1 \mathrm{M}$ HEPES, $\mathrm{pH} 7.8$ and $15 \%$ PEG 4000 , with a SlrP/Trx 1 molar ratio of $1: 2$ by mixing $216 \mu \mathrm{M}$ SlrP with $430 \mu \mathrm{M}$ Trx 1 . The crystals were flash frozen in liquid nitrogen using the crystallization solution supplemented with $30 \%$ ethylene glycol as cryoprotectant. A native diffraction data set was collected at $100 \mathrm{~K}$ on beamline ID29 at synchrotron ESRF (Grenoble, France). The crystals diffracted up to $3.3 \AA$ resolution in space group $\mathrm{P} 2{ }_{1} 2_{1} 2_{1}$. The diffraction data were processed using the XDS package [29]. Molecular replacement performed using the program PHASER [30] failed to solve the crystallographic phase problem by using either of the LRR and NEL domains from the available structures of IpaH3 (PDB ID 3CVR) [21] or SspH2 (PDB ID 3G06) [22].

New co-crystals of the SlrP/Trx 1 complex were obtained using a seleno-methionine-labelled form of SlrP. SeMet-SlrP at $274 \mu \mathrm{M}$ was co-crystallized with $609 \mu \mathrm{M}$ Trx 1 in a crystallization solution containing $18 \%$ PEG 8000, 0.2M Mg Acetate and $0.1 \mathrm{M}$ Tris- $\mathrm{HCl}, \mathrm{pH} 8.8$. The crystals were flash frozen in liquid nitrogen using the crystallization solution supplemented with $30 \%$ glycerol as cryoprotectant. A data set was collected at the selenium edge on beamline Proxima 1 of the synchrotron SOLEIL (Gif-sur-Yvette, France). The energy of the beamline was set to correspond to the maximum of the anomalous factor $\mathrm{f}^{\prime}$ ' as determined by a fluorescence scan. The crystals diffracted up to $3.6 \AA$ and were isomorphous to the native ones. The sub-structure determination was performed with the program SHELXD [31] and initial phasing was performed with the program PHASER [30]. Electron density modification was carried out using PARROT [32]. Subsequent model building was done manually with COOT [33]. Non-crystallographic symmetry was applied in the first steps of refinement performed against the SAD data set using BUSTER [34]. Final refinement was performed against the native data set using PHENIX [35]. The data processing and refinement statistics are given in Table 1.

\section{Ubiquitination assays}

Single (K8A, K36A and K94A) and the triple (K8A/K36A/K94A) derivative mutant versions of Trx 1 were constructed and tested in ubiquitination assays following the protocol previously published [24] with some modifications. Briefly, ubiquitination reactions were performed in a $20-\mu 1$ mixture containing buffer A (25 mM Tris- $\mathrm{HCl}, \mathrm{pH} 7.5,50 \mathrm{mM} \mathrm{NaCl}, 5 \mathrm{mM}$ ATP, $10 \mathrm{mM} \mathrm{MgCl}, 0.1 \mathrm{mM}$ DTT), $1 \mu \mathrm{g}$ of HAtagged ubiquitin (Boston Biochem), 0,25 $\mu \mathrm{g}$ of human recombinant E1 (Boston Biochem), and $1 \mu \mathrm{g}$ of E2 (human recombinant UbcH5b from Boston Biochem) in the presence of $0.5 \mu \mathrm{g}$ of GST-SlrP and/or GSTTrx1 (wild type or mutants). Reactions were incubated at $37^{\circ} \mathrm{C}$ for $1 \mathrm{~h}$ and stopped by addition of an equal volume of Laemmli sample buffer containing $100 \mathrm{mM}$ DTT. Reaction mixtures were immunoprecipitated with agarose conjugate anti-Trx polyclonal antibody (Santa Cruz Biotechnology) and then subjected to immunoblot analysis.

\section{Structure analysis}

Structure comparisons and calculations of rmsd distances have been performed by the protein structure comparison service Fold at the European Bioinformatics Institute [36]. Contact and interface calculations have been performed by the Protein Interfaces, Surfaces and Assemblies service (PISA) at the European Bioinformatics Institute [37]. Multiple sequence alignment was performed using ClustalW [38] and secondary structure elements were shown at the top of the alignment using ESPript [39]. The model of the apo dimer of SlrP was prepared using COOT. Figures of 3D structures were generated using PYMOL (DeLano Scientific LLC). The final model of the native SlrP/Trx1 complex was evaluated using the COOT validation tools, and the MolProbity software [40] before deposition in the Protein Data Bank (Berman et al, 2000) under ID code 4PUF. 


\section{RESULTS}

\section{Crystal structure of the SIrP/Trx1 complex}

LRR-NEL effectors present at their N-terminus a third domain that has been shown to direct their translocation [41] and localization in the host cell [42]. There is a substantial amount of evidence suggesting that secretion signals of T3SS effectors are intrinsically disordered and that this structural property could help recognition and transport by the secretion systems [43]. In order to facilitate the crystallization process, we removed the $139 \mathrm{~N}$-terminal residues corresponding to the predicted translocation signal of SlrP [41]. Because T3SS signal sequences are reported as non-cleaved in vivo [44], we verified that the truncated His-tagged recombinant protein restricted to the LRR and NEL domains of SlrP (residues 140-765, 73kDa) kept its ability to bind Trx1 (Figure S1). Pull-down experiments confirmed that the truncated form of SlrP could be used for co-crystallization experiments with Trx1.

We obtained crystals containing two SlrP and two Trx 1 molecules per asymmetric unit. Trx 1 displays the well-characterized $\alpha / \beta$ fold very similar to the reduced form of free Trx1 (PDB ID 1ERT) [45], with an rmsd distance of about $0.67 \AA$ for $105 \mathrm{C} \alpha$ atoms aligned between the two subunits. The two SlrP subunits present the same overall structure (Figure 1A) with an rmsd distance of $1.1 \AA$ for $589 \mathrm{C} \alpha$ atoms aligned. The LRR domain of SlrP (residues 147-450) is N-capped by two antiparallel $\alpha$-helices, $\alpha 1$ and $\alpha 2$, and contains 13 LRR motifs (Figure 1B). The loop of LRR-11 contains a short extension. The loop of LRR-12 is replaced by a capping $\alpha$-helix, $\alpha 3$, while the loop of LRR-13 forms a linker of 15 residues (451-464) between the LRR domain and the fully $\alpha$-helical NEL domain (helices $\alpha \mathrm{A}$ to $\alpha \mathrm{N}$, residues 465765). The later displays the characteristic globular core followed by a protruding helix bundle (Figure 1A). In both copies of the NEL domain there was no visible electron density for loop $\alpha \mathrm{M}-\alpha \mathrm{N}$ (residues 709728) of the C-terminal helix bundle. In contrast, the loop linking the LRR and NEL domains that was disordered in the published apo structures of IpaH3 [21] and SspH2 [22] is clearly defined in the Trx1 bound SlrP structure. The catalytic loop carrying the conserved $\mathrm{C}_{546} \mathrm{XD}_{548}$ motif [24] is exposed to the solvent and accessible to the E2-Ub conjugate.

\section{The SIrP/Trx1 complex forms a heterotetramer}

Two head-to-tail SlrP subunits are bridged by two Trx1 subunits, resulting in an unexpected heterotetramer (Figure 2A). The latter forms a stable complex characterized by a total buried solventaccessible surface area of $5208.2 \AA^{2}$ and a solvation free energy gain $\Delta^{\mathrm{i}} \mathrm{G}$ of $-21.3 \mathrm{kcal} / \mathrm{mol}$.

The two bound Trx 1 subunits make only few contacts with each other, involving a small interface area of $125.7 \AA^{2}\left(\Delta^{\mathrm{i}} \mathrm{G}=-0.3 \mathrm{kcal} / \mathrm{mol}\right)$ at their $\mathrm{C}$-terminal helices (Figure $2 \mathrm{~A}$ ). This Trx1-Trx1 interaction mode is completely different from that observed in the inactive oxidized Trx1 dimer (PDB ID 1AIU) in which monomers are disulfide-linked through Cys73 from each subunit [45]. Similarly, the two SlrP subunits display a very small interface area of $96.1 \AA^{2}\left(\Delta^{\mathrm{i}} \mathrm{G}=+0.3 \mathrm{kcal} / \mathrm{mol}\right)$ involving a small loop located between the LRR-11 and LRR-12 motifs (residues $\mathrm{P}_{415}-\mathrm{Q}_{416}-\mathrm{P}_{417}$ ) (Figure 2B). The contacts between SlrP and Trx 1 subunits are slightly more important. Each SlrP molecule interacts with both Trx 1 subunits. The concave surface of the LRR domain forms the so-called type-I binding site (Figure 2C) characterized by an average interface area of $729.2 \AA^{2}$ and a solvation energy gain of $\Delta^{\mathrm{i}} \mathrm{G}=-2.6 \mathrm{kcal} / \mathrm{mol}$. These contacts mainly involve basic residues from the SlrP LRR domain and the acidic region Glu56-Asp61 from Trx1. The linker loop of SlrP (residues 451-464) forms a type-II binding site (Figure 2D) characterised by an average interface area of about the same size $\left(655.5 \AA^{2}\right)$ but associated with a larger solvation free energy gain of $\Delta^{\mathrm{i}} \mathrm{G}=-10.9 \mathrm{kcal} / \mathrm{mol}$. These contacts mainly involve residues from the linker region of SlrP and loops 68-74 and 88-92 from Trx1. Analysis of the heterotetramer interfaces using PDBePISA suggested that only this type-II Slrp/Trx1 interface could form stable assemblies in solution.

Detailed analysis of this type-II interface (Figure 2D) suggested that mutating into glutamate the SlrP hydrophobic residues Val459 and Val461 or the Trx1 residue Gly91 could disrupt the complex. This hypothesis was experimentally tested using the yeast two-hybrid system. As seen in Figure 3, whereas wild-type SlrP and Trx1 were able to interact, mutant SlrP-V459E/V461E was unable to bind Trx1, and 
mutant Trx 1-G91E was unable to bind SlrP. These results confirm the importance of the type-II SlrP/Trx1 interface for the interaction of the two proteins.

The question of the biological relevance of the heterotetramer observed in the crystal, with formation of the type-I SlrP/Trx1 interface, was assessed using SEC-MALLS analysis (Figure 4). SlrP was eluted in two peaks corresponding to a monomer and a dimer, respectively. Trx1 was eluted as a monomer. An equimolar mix of SlrP and Trx1 was eluted in two peaks corresponding to a 1:1 complex and a 2:2 complex. This result demonstrates that two forms of SlrP, monomeric and dimeric, co-exist in solution and that both of them bind Trx 1 .

\section{Identification of a Trx1 ubiquitination site}

The catalytic loop of the SlrP subunits does not directly interact with a bound substrate molecule, and none of the 12 lysines contained in Trx 1 was clearly orientated toward the active site of the NEL domain, suggesting that the observed heterotetramer could correspond to an intermediate in the ubiquitination mechanism.

Three Trx1 lysine residues (Lys8, Lys36 and Lys94) exposed at the surface of the molecule (Figure $5 \mathrm{~A}$ ) were considered as potential ubiquitination sites and mutated into alanine. The ability of SlrP to ubiquitinate these mutant proteins was tested in vitro in the presence of recombinant human E1 ubiquitinactivating enzyme and E2 ubiquitin-conjugating enzyme. The ubiquitination profiles of Trx 1-K8A and Trx1-K36A mutants were similar to the wt-Trx1 profile. Interestingly, Trx1-K94A and the triple mutant protein Trx1-K8A/K36A/K94A displayed a strongly reduced ubiquitination profile (Figure 5B). These results suggested that Lys94 is one of the main target sites for Trx1 SlrP-dependent ubiquitination. However, the ubiquitination profiles of Trx1-K94A and of the triple Trx 1 mutant still displayed some slight bands in the Western blot analysis and it cannot be excluded that other lysine residues could also be ubiquitinated. The Trx 1 residue Lys94 and the SlrP catalytic Cys546 are spaced about 40 $\AA$ apart (Figure $5 \mathrm{~A})$. The SlrP catalytic loop is exposed to the solvent, suggesting that it is compatible with the binding of the E2-Ub complex. However, an important reorientation of the NEL domain will be required after ubiquitin binding to allow Lys94 to exert a nucleophilic attack on the intermediate Cys546-ubiquitin thioester bond. Once the ubiquitin molecule transferred to Trx1, this post-translational modification would most probably impair the conserved $\mathrm{C}_{32} \mathrm{GPC}_{35}$ catalytic motif, located close to Lys36 on the same face of Trx1 than the Lys94 ubiquitination site (Figure 5A), to interact with the Trx1 substrates. This would explain the observed SlrP-dependent inhibition of the Trx1 reducing activity [24]. Interestingly, other post-translational modifications including glutathionylation, thiol-oxidation, and S-nitrosylation, at the non-active cysteines have already been shown to importantly contribute to the regulation of Trx1 [46].

\section{DISCUSSION}

Previous studies demonstrated that removal of the LRR domain of SlrP (residues 1-457) resulted in dramatic increase of its ubiquitin E3 ligase activity [22]. These results suggest that the LRR domain, known as a versatile structural framework for protein-protein interaction [47], is not implicated in substrate binding but is rather involved in the regulation of the adjacent NEL catalytic domain [14].

The structure of the SlrP/Trx 1 complex presented in this paper demonstrates the linker region between the NEL and LRR domains plays an essential role in substrate binding. Comparison of the SlrP/Trx 1 complex with the apo structures of the NEL effectors IpaH3 from Shigella (PDB ID 3CVR) [21] and SspH2 from Salmonella (PDB ID 3G06) [22] shed light on the molecular mechanism of these ubiquitin E3 ligases.

Holo SlrP displays the same open conformation as the apo structure of IpaH3 (Figure 6A). An internal LRR-NEL interface area of about $711.2 \AA^{2}\left(\Delta^{\mathrm{i}} \mathrm{G}=-3.4 \mathrm{kcal} / \mathrm{mol}\right)$ stabilizes the relative orientation of the LRR and NEL domains. As observed in IpaH3 [21], a conserved phenylalanine residue from loop $\alpha \mathrm{G}-\alpha \mathrm{H}$ in the NEL domain, corresponding to SlrP Phe604 (Figure S2), is buried into a hydrophobic 
pocket formed by the $\alpha$-helix capping the C-terminus of the LRR-domain (SlrP helix $\alpha 3$ ) and the convex side of this LRR domain (Figure 6B).

In the SspH2 structure (PDB ID 3G06) [22], the SspH2 residue Leu638 corresponding to SlrP Phe604 (Figure S2) is buried in a hydrophobic pocket formed by the other face of the $\alpha$-helix C-capping the LLR domain ( $\mathrm{SspH} 2$ helix $\alpha 4=\mathrm{SlrP}$ helix $\alpha 3$ ) and the N-terminal extremity of the linker loop. In this closed conformation, the NEL domain is turned toward the concave face of the LRR domain and the catalytic cysteine is buried in this interface (Figure 6C). Interestingly, it has been shown that the conservative Leu to Phe mutation of the essential hydrophobic residue from loop $\alpha \mathrm{G}-\alpha \mathrm{H}$ had no effect on the LRRdependent inhibition [23]. Furthermore, the hydrophobic residues (Ile479, Phe481 and Met483) from the $\mathrm{SspH} 2$ linker region that have been shown to stabilize this conformation are conserved in SirP (Val449, Phe451 and Met453, Figure S2). This suggests that SlrP could adopt the SspH2 open conformation. However, this conformation is incompatible with the Trx1 type-II binding mode (Figure 6D).

Together with the observation of an equilibrium between a monomeric and a dimeric form of apo SlrP in solution (Figure 4A), these data further suggest that apo SlrP could fluctuate between two monomeric conformations: (i) an auto-inhibited closed conformation that sequesters the catalytic cysteine and masks the substrate-binding site, and (ii) an open conformation compatible with substrate binding and interaction with the E2-Ub complex. Formation of the Ub-E3 thioester bond [23] could induce the release of the inhibitory interaction between the NEL residue Phe604 and the convex side of the LRR domain, thus allowing a conformational change compatible with the transfer of the ubiquitin molecule to Trx 1 Lys94. This step could involve internal reorganization of the NEL domain that has been shown to contain three subdomains [19]. The catalytic cysteine is indeed situated in the loop connecting the N-terminal lobe to the middle lobe. Its thioesterification with ubiquitin could thus modify the relative orientation of these two subdomains and disrupt the autoinhibiting interaction between the LRR domain and the hydrophobic NEL residue Phe604 from loop $\alpha \mathrm{G}-\alpha \mathrm{H}$ located in the middle lobe.

We propose that the apo SlrP dimer observed in solution (Figure 4A) could form between two subunits in the closed conformation. In the crystallized heterotetramer, the contacts between the two SlrP subunits in the open conformation are indeed too weak (Figure 2B) to form a stable assembly in the absence of bound Trx1. The head-to-tail dimerization mode observed in the heterotretramer could be conserved and the contacts made by the two Trx 1 subunits could be made by the NEL domains that superimpose in the closed conformation with the Trx 1 molecules bound to the open conformation (Figure 6D). The 2:2 SlrP/Trx1 complex observed in solution (Figure 4C) could thus form either by head-to-tail dimerization of two type-II SlrP/Trx 1 1:1 complexes or by direct substrate binding to the apo SlrP dimer.

Finally, the Trx 1 ubiquitinatable residue Lys94 is buried in the resulting type-I interface, suggesting that Trx1 ubiquitination would anyway require dissociation of the heterotetramer into two type-II SlrP/Trx 1 complexes. The conformational change of induced upon thioesterification of the catalystic Cys546 would thus most probably induce dissociation of the two type-II SlrP/Trx1 complexes.

This proposed molecular mechanism (Figure 7) could apply to other members of the NEL family. However, multiple sequence alignments performed with distant homologues of the LRR-NEL family of T3SS effectors (Figure S2) demonstrated that the $\mathrm{P}_{415}-\mathrm{Q}_{416}-\mathrm{P}_{417}$ motif forming the small SlrP-SlrP contact region of the heterotetramer corresponds to a small extension restricted to SlrP members of the family. This suggests that other LRR-NEL effectors could not form dimers. SlrP dimerization could be required to stabilize binding of the small molecule substrate Trx1, which presents only a small interface area. Recently, the structural analysis of the LRR domain of SspH1 in complex with the small HR1B domain of PKN1 (PDB ID 4NKG) [48] suggested a totally distinct substrate-binding mode. However only a small domain of the protein substrate (residues 122-199 from 942) is present in this structure and we cannot exclude that binding of full-length PKN1 to full-length SspH1 would involve other parts of the proteins, and in particular the linker region as observed in the SlrP/Trx1 complex. Another structure of a LRR-NEL effector in complex with its full-length protein substrate will be required to elucidate if the substratebinding mode is specific to each family member. 


\section{ACKNOWLEDGMENTS}

We thank staffs of beamlines Proxima-1 at the French synchrotron SOLEIL and ID14, ID23 and ID29 at the European Synchrotron Radiation Facility. We acknowledge the use of the IMAGIF crystallization platform and thank Pr. H. van Tilbeurgh for critical reading and correction of the manuscript.

\section{FUNDINGS}

This work was supported by grant P08-CVI-03487, from the Consejería de Economía, Innovación y Ciencia, Junta de Andalucía, Spain, grant SAF2010-15015, from the Spanish Ministry of Economy and Competitiveness and the European Regional Development Fund, grant FR2009-0103 from the Spanish Ministry of Science and Innovation and the programme Picasso-2010 from the Partenariat Hubert Curien. 


\section{REFERENCES}

1. Ghosh, P. (2004) Process of protein transport by the type III secretion system. Microbiol. Mol. Biol. Rev. 68, 771-795

2. Duncan, M.C, Linington, R. G. and Auerbuch, V. (2012) Chemical inhibitors of the type three secretion system: disarming bacterial pathogens. Antimicrob. Agents Chemother. 56, 5433-5441

3. Agbor, T. A. and Mc Cormick, B. A. (2011) Salmonella effectors: important players modulating host cell function during infection. Cell. Microbiol. 13, 1858-1869

4. Ramos-Morales, F. (2012) Impact of Salmonella enterica Type III Secretion System Effectors on the Eukaryotic Host Cell. ISRN Cell Biol 2012

5. Ogawa, M., Handa, Y., Ashida, H., Suzuki, M. and Sasakawa, C. (2008) The versatility of Shigella effectors. Nat. Rev. Microbiol. 6, 11-16

6. Shao, F. (2008) Biochemical functions of Yersinia type III effectors. Curr. Opin. Microbiol. 11, 21-29

7. van der Heijden, J. and Finlay, B. B. (2012) Type III effector-mediated processes in Salmonella infection. Future Microbiol. 7, 685-703

8. Angot, A., Vergunst, A., Genin, S. and Peeters, N. (2007) Exploitation of eukaryotic ubiquitin signaling pathways by effectors translocated by bacterial type III and type IV secretion systems. PLoS Pathog. 3, e3

9. Rytkonen, A. and Holden, D. W. (2007) Bacterial interference of ubiquitination and deubiquitination. Cell Host Microbe 1, 13-22

10. Sun, L. and Chen, Z. J. (2004) The novel functions of ubiquitination in signaling. Curr. Opin. Cell. Biol. 16, 119-126

11. Kerscher, O., Felberbaum, R. and Hochstrasser, M. (2006) Modification of proteins by ubiquitin and ubiquitin-like proteins. Annu. Rev. Cell. Dev. Biol. 22, 159-180

12. Metzger, M. B., Hristova, V. A. and Weissman, A. M. (2012) HECT and RING finger families of E3 ubiquitin ligases at a glance. J. Cell. Sci. 125, 531-537

13. Budhidarmo, R., Nakatani, Y. and Day, C. L. (2012) RINGs hold the key to ubiquitin transfer. Trends Biochem. Sci. 37, 58-65

14. Hicks, S. W. and Galan, J. E. (2010) Hijacking the host ubiquitin pathway: structural strategies of bacterial E3 ubiquitin ligases. Curr. Opin. Microbiol. 13, 41-46

15. Janjusevic, R., Abramovitch, R. B., Martin, G. B. and Stebbins, C. E. (2006) A bacterial inhibitor of host programmed cell death defenses is an E3 ubiquitin ligase. Science 311, 222-226

16. Diao, J., Zhang, Y., Huibregtse, J. M., Zhou, D. and Chen, J. (2008) Crystal structure of SopA, a Salmonella effector protein mimicking a eukaryotic ubiquitin ligase. Nat. Struct. Mol. Biol. 15, 6570

17. Rohde, J. R., Breitkreutz, A., Chenal, A., Sansonetti, P. J. and Parsot, C. (2007) Type III secretion effectors of the IpaH family are E3 ubiquitin ligases. Cell Host Microbe 1,77-83

18. Bella, J., Hindle, K. L., McEwan, P. A. and Lovell, S. C. (2008) The leucine-rich repeat structure. Cell. Mol. Life Sci. 65, 2307-2333

19. Singer, A. U., Rohde, J. R., Lam, R., Skarina, T., Kagan, O, Dileo, R., Chirgadze, N. Y., Cuff, M. E. Joachimiak, A., Tyers, M., Sansonetti, P. J., Parsot, C. and Savchenko, A. (2008) Structure of the Shigella T3SS effector IpaH defines a new class of E3 ubiquitin ligases. Nat. Struct. Mol. Biol. 15, 1293-1301

20. Scheffner, M. and Staub. O. (2007) HECT E3s and human disease. BMC Biochem. 8 Suppl 1, S6

21. Zhu, Y., Li, H., Hu, L., Wang, J., Zhou, Y., Pang, Z., Liu, L. and Shao, F. (2008) Structure of a Shigella effector reveals a new class of ubiquitin ligases. Nat. Struct. Mol. Biol. 15, 1302-1308

22. Quezada, C. M., Hicks, S. W., Galan, J. E. and Stebbins, C. E. (2009) A family of Salmonella virulence factors functions as a distinct class of autoregulated E3 ubiquitin ligases. Proc. Natl. Acad. Sci. U. S. A. 106, 4864-4869

23. Chou, Y. C., Keszei, A. F., Rohde, J. R., Tyers, M. and Sicheri, F. (2012) Conserved structural mechanisms for autoinhibition in IpaH ubiquitin ligases. J. Biol. Chem. 287, 268-275 
24. Bernal-Bayard, J. and Ramos-Morales, F. (2009) Salmonella type III secretion effector SlrP is an E3 ubiquitin ligase for mammalian thioredoxin. J. Biol. Chem. 284, 27587-27595

25. Hanahan, D. (1983) Studies on transformation of Escherichia coli with plasmids. J. Mol. Biol. 166, 557-580

26. Bullock, W. O., Fernandez, J. M. and Short, J. M. (1987) XL1-BLUE: a high efficiency plasmid transforming RecA Escherichia coli strain with beta-galactosidase selection. Biotechniques 5, 376379

27. Harper, J. W., Adami, G. R., Wei, N., Keyomarsi, K. and Elledge, S. J. (1993) The p21 Cdkinteracting protein Cip 1 is a potent inhibitor of G1 cyclin-dependent kinases. Cel1 75, 805-816

28. Sherman, F., Fink, G. R. and Hicks, J. B. (1986) Laboratory course manual for methods in yeast genetics. Cold Spring Harbor Laboratory, Cold Srping Harbor, NY.

29. Kabsch, W. (2010) Xds. Acta Crystallogr. D Biol. Crystallogr. 66, 125-132

30. McCoy, A. J., Grosse-Kunstleve, R. W., Adams, P. D., Winn, M. D., Storoni, L. C. and Read, R.J. (2007) Phaser crystallographic software. J. Appl. Cryst. 40, 658-674

31. Sheldrick, G. M. (2008) A short history of SHELX. Acta Crystallogr. A 64, 112-122

32. Zhang, K. Y., Cowtan, K. and Main, P. (1997) Combining constraints for electron-density modification. Methods Enzymol. 277, 53-64

33. Emsley, P. and Cowtan, K. (2004) Coot: model-building tools for molecular graphics. Acta Cryst. D Biol. Cryst. 60, 2126-2132

34. Bricogne, G., Blanc. E., Brandl, M., Flensburg, C., Keller, P., Paciorek, W., Roversi, P., Smart, O.S. , Vonrhein, C. and Womack, T.O. (2009) BUSTER. version 280 Cambridge, United Kingdom: Global Phasing Ltd.

35. Afonine, P.V., Grosse-Kunstleve, R. W., Echols, N., Headd, J.J., Moriarty, N. W., Mustyakimov, M., Terwilliger, T. C., Urzhumtsev, A. Zwart, P. H. and Adams, P. D. (2012) Towards automated crystallographic structure refinement with phenix.refine. Acta Crystallogr. D Biol. Crystallogr. 68, 352-367

36. Krissinel, E. and Henrick, K. (2004) Secondary-structure matching (SSM), a new tool for fast protein structure alignment in three dimensions. Acta Crystallogr. D Biol. Crystallogr. 60, 2256-2268

37. Krissinel, E. and Henrick, K. (2007) Inference of macromolecular assemblies from crystalline state. J. Mol. Biol. 372, 774-797

38. Chenna, R., Sugawara, H., Koike, T., Lopez, R., Gibson TJ, Higgins, D. G. and Thompson, J. D. (2003) Multiple sequence alignment with the Clustal series of programs. Nucleic Acids Res. 31, 3497-3500

39. Gouet, P., Robert, X. and Courcelle, E. (2003) ESPript/ENDscript: Extracting and rendering sequence and 3D information from atomic structures of proteins. Nucleic Acids Res. 31, 3320-3323

40. Chen, V. B., Arendall, W. B., 3rd, Headd, J. J., Keedy, D. A., Immormino, R. M., Kapral, G. J., Murray, L. W., Richardson, J. S. and Richardson, D. C. (2010) MolProbity: all-atom structure validation for macromolecular erystallography. Acta Crystallogr. D Biol. Crystallogr. 66, 12-21

41. Miao, E. A. and Miller, S. I (2000) A conserved amino acid sequence directing intracellular type III secretion by Salmonella typhimurium. Proc. Natl. Acad. Sci. U. S. A. 97, 7539-7544

42. Miao, E. A., Brittnacher, M., Haraga, A., Jeng, R. L., Welch, M. D. and Miller, S. I. (2003) Salmonella effectors translocated across the vacuolar membrane interact with the actin cytoskeleton. Mol. Microbiol. 48, 401-415

43. Buchko, G. W., Niemann, G., Baker, E. S., Belov, M. E., Smith, R. D., Heffron, F., Adkins, J. N. and McDermott, J. E. (2010) A multi-pronged search for a common structural motif in the secretion signal of Salmonella enterica serovar Typhimurium type III effector proteins. Mol. Biosyst. 6, 24482458

44. Mota, L. J., Sorg, I. and Cornelis, G. R. (2005) Type III secretion: the bacteria-eukaryotic cell express. FEMS Microbiol. Lett. 252, 1-10 
45. Weichsel, A., Gasdaska, J. R., Powis, G. and Montfort, W. R. (1996) Crystal structures of reduced, oxidized, and mutated human thioredoxins: evidence for a regulatory homodimer. Structure 4, 735751

46. Haendeler, J. (2006) Thioredoxin-1 and posttranslational modifications. Antioxid. Redox. Signal. 8, $1723-1728$

47. Kobe, B. and Kajava, A. V. (2001) The leucine-rich repeat as a protein recognition motif. Curr. Opin. Struct. Biol. 11, 725-732

48. Keszei, A. F., Tang, X., McCormick, C., Zeqiraj, E., Rohde, J. R., Tyers, M. and Sicheri, F. (2013) Structure of an SspH1-PKN1 complex reveals the basis for host substrate recognition and mechanism of activation for a bacterial E3 ubiquitin ligase. Mol. Cell. Biol. 34, 362-373 


\section{FIGURE LEGENDS}

\section{Figure 1: Structure of SIrP}

(A) Overall 3D structure of the SlrP subunit. The polypeptide chain is shown as cartoon coloured from blue (N-ter) to red (C-ter). The secondary structure elements are labelled as well as the LRR-domain (residues 147-450), the linker region (residues 451-464) and the NEL-domain (residues (465-765). (B) Sequence alignment of the 13 LRR repeats. LRR-1 (residues 180-199), LRR-2 (residues 200-220), LRR-3 (residues 221-242), LRR-4 (residues 243-262), LRR-5 (residues 263-284), LRR-6 (residues 285-305), LRR-7 (residues 306-325), LRR-8 (residues 326-346), LRR-9 (residues 347-368), LRR-10 (residues 369389 ) and LRR-11 (residues 390-417) are aligned with the positions of the conserved $\beta$-strand displayed at the top of the alignment. The unconventional LRR-12 (residues 418-445) and LRR-13 (residues 446-464) are shown separately with the position of the capping helix $\alpha 3$ and the linker region highlighted above the corresponding sequence.

\section{Figure 2: The SIrP/Trx1 heterotetramer}

(A) Crystal structure of the head to tail SlrP/Trx1 heterotetramer. Each subunit is shown as cartoon in the envelope corresponding to the surface of the complex. The SlrP molecules are coloured by spectrum from blue to red while the Trx 1 molecules are coloured gray and beige, respectively. (B) The SlrP/SlrP interface. Close view of the contact region between the two SlrP subunits. Residues $\mathrm{P}_{415}-\mathrm{Q}_{416}-\mathrm{P}_{417}$ from the LRR-11 extension are highlighted. (C) Type-I SlrP/Trx1 interface. Close view of the contact region between the LRR domain of SlrP and one molecule of Trx1. Residues directly involved in the contact are highlighted. (D) Type-II SlrP/Trx1 interface. Close view of the contact region between the linker loop of SlrP and the second molecule of Trx1. Residues directly involved in the contact are highlighted. SlrP residues Val459 and Val461 as well as Trx1 Gly91 have been mutated into E to assess the biological relevance of this interaction mode.

\section{Figure 3: Disruption of binding between SIrP and Trx1 by point mutations in the type-II SIrP/Trx1} interface.

The yeast two-hybrid system was used to test the interaction between SlrP, Trx1 and their mutants. Diploids were obtained by conjugation between yeast strain Y2HGold containing derivatives of pGBKT7, and yeast strain Y187 containing derivatives of pGADT7, as indicated. The pGBKT7 vector expresses proteins fused to the Gal4 DNA-binding domain, whereas pGADT7 expresses proteins fused to the Gal4 activation domain. The interaction between the two hybrid proteins is shown by growth in the absence of histidine and adenine. Empty vectors $(-)$ were used as negative controls.

\section{Figure 4: SEC-MALLS analysis of the SIrP/Trx1 interaction.}

(A) An SlrP sample at $1.2 \mathrm{mg} / \mathrm{ml}(16 \mu \mathrm{M})$ eluted from the column in two peaks corresponding to a monomer $(79 \mathrm{kDa})$ and a dimer $(149 \mathrm{kDa})$, respectively. (B) A Trx 1 sample at $1.0 \mathrm{mg} / \mathrm{ml}(83 \mu \mathrm{M})$ eluted from the column as a monomer $(12 \mathrm{kDa})$. (C) An equimolar mix of SlrP and Trx1 eluted from the column in two peaks corresponding to a 1:1 complex $(86 \mathrm{kDa})$ and a 2:2 complex $(172 \mathrm{kDa})$, respectively.

\section{Figure 5: Trx1 ubiquitination}

(A) Localisation of the three Trx1 mutated residues Lys8, Lys36 and Lys94. The distance between SlrP catalytic Cys546 and Trx1 ubiquitinated Lys94 is indicated. (B) SlrP-dependent ubiquitination assays using the Trx1-K8A, -K36A, -K94A and -K8A/K36A/K94A mutants. Reactions performed in the presence of HA-ubiquitin, E1, E2, GST-SlrP (SlrP), and GST-Trx1 (Trx), as indicated, were immunoprecipitated with anti-Trx antibodies. The precipitates were resolved in 10\% SDS-PAGE and analyzed by immunoblot with anti-HA (upper panel) or anti-Trx (lower panel) antibodies. Putative ubiquitinated forms of Trx 1 are indicated (Trx-Ub). 
Figure 6: Comparison with other LRR-NEL effectors

(A) Superimposition of the NEL domains. One SlrP subunit of the SlrP/Trx1 complex is shown as cartoon coloured in yellow. Apo IpaH3 (PDB ID 3CVR) is shown in magenta and apo SspH2 (PDB ID 3G06) in cyan. (B) Close view of the conserved NEL-LRR contacts in the open conformation observed in the SlrP/Trx1 complex. SlrP is shown as cartoon coloured spectrum with the electrostatic surface of the LRR domain in transparency. The NEL residue Phe604 from loop $\alpha \mathrm{G}-\alpha \mathrm{H}$ is buried in a hydrophobic pocket on the convex side of the LRR domain. The SlrP catalytic cysteine Cys546 is highlighted. (C) Close view of the NEL-LRR contacts in the closed conformation observed in the SspH2 structure (PDB ID 3G06). $\mathrm{SspH} 2$ is shown as cartoon coloured spectrum with the electrostatic surface of the LRR domain in transparency. SspH2 residue Leu638 from loop $\alpha \mathrm{G}-\alpha \mathrm{H}$ (equivalent to SlrP residue Phe604) is buried in a hydrophobic pocket on the concace side of the LRR domain. The $\mathrm{SspH} 2$ catalytic cysteine is highlighted. (D) Superimposition of the LRR domain of $\mathrm{SspH} 2$ with a SlrP/Trx1 heterodimer. The NEL domain of apo $\mathrm{SspH} 2$ (PDB ID 3G06, in cyan) occupies the same position as the Trx1 molecule (in beige) bound in the type-I binding site of SlrP (in yellow).

Figure 7: Schematic representation of the proposed molecular mechanism. The equilibrium between a monomeric and a dimeric conformation for is inferred from the SEC-MALLS analysis. The equilibrium between the open and closed monomeric conformation of apo SlrP is inferred from the comparison with the other members of the family. The 2:2 SlrP/Trx 1 complex corresponds to the structure presented in this article. The 1:1 complex conformation is based on the type-II SlrP/Trx 1 interaction mode. The different steps allowing thioesterification of the SlrP catalytic C546 by the human E2-Ub complex are still unclear. But dissociation of the 2:2 complex and conformational change of the of the 1:1 complex required for ubiquitin transfer from SlrP Cys546 to Trx1 Lys94 are represented. 
Table 1: X-ray data processing and refinement statistics.

\begin{tabular}{|c|c|c|}
\hline Data processing statistics & Native data set & SAD data set (SeMet) \\
\hline Space group & $\mathrm{P} 22_{1} 2_{1}$ & $\mathrm{P} 22_{1} 2_{1}$ \\
\hline Unit-cell parameters $(\AA)$ & $\mathrm{a}=106.3 ; \mathrm{b}=134.8 ; \mathrm{c}=154.6$ & $a=106.7 ; b=135.0 ; c=153.9$ \\
\hline Unit-cell angles $\left(^{\circ}\right)$ & $\alpha=90^{\circ} ; \beta=90^{\circ} ; \gamma=90^{\circ}$ & $\alpha=90^{\circ} ; \beta=90^{\circ} ; \gamma=90^{\circ}$ \\
\hline Resolution range $(\AA)$ & $45.0-3.3(3.49-3.29)^{*}$ & $50.0-3.6(3.84-3.63)^{*}$ \\
\hline No. of unique reflections & $34042(5402)$ & $48641(7789)$ \\
\hline Completeness (\%) & $99.4(98.1)$ & $99.8(98.8)$ \\
\hline Redundancy & $4.4(4.6)$ & $7.8(7.7)$ \\
\hline Mean $\mathrm{I} / \sigma(\mathrm{I})$ & $16.84(2.83)$ & $15.77(3.48)$ \\
\hline $\mathrm{R}_{\text {meas }}(\%)^{\dagger}$ & $5.6(69.9)$ & $9.8(62.9)$ \\
\hline Refinement statistics & Against native data set & $Y \longdiv { 2 }$ \\
\hline Resolution range & $44.0-3.3(3.4-3.3)$ & $\gamma$ \\
\hline No. of molecules/a.u. & 4 & \\
\hline $\mathrm{R}_{\text {work }}(\%)^{\ddagger}$ & $27.17(43.54)$ & \\
\hline $\mathrm{R}_{\text {free }}(\%)^{\S}$ & $30.84(47.99)$ & \\
\hline \multicolumn{2}{|l|}{ Ramachandran } & \\
\hline Favoured $(\%)$ & 91.4 & \\
\hline Disallowed (\%) & 0.9 & \\
\hline \multicolumn{2}{|l|}{ R.M.S.D. } & \\
\hline Bond lengths $(\AA)$ & 0.003 & \\
\hline Bond angles $\left({ }^{\circ}\right)$ & 0.785 & \\
\hline Dihedral angle $\left({ }^{\circ}\right)$ & 11.736 & \\
\hline Chirality & 0.049 & \\
\hline Planarity & 0.005 & \\
\hline Mean B value $\left(\AA^{2}\right)$ & 118.53 & \\
\hline
\end{tabular}

* Numbers in parentheses represent values in the highest resolution shell.

$\dagger \mathrm{R}_{\text {meas }}=\operatorname{Shkl}[\mathrm{N} / \mathrm{N}-1]^{1 / 2} \mathrm{Si}|\mathrm{Ii}(\mathrm{hkl})-<\mathrm{I}(\mathrm{hkl})>| / \mathrm{ShklSi} \mathrm{Ii}(\mathrm{hkl})$ where $\mathrm{N}$ is the multiplicity of a given reflection, Ii(hkl) is the integrated intensity of a given reflection and $\langle\mathrm{I}(\mathrm{hkl})\rangle$ is the mean intensity of multiple corresponding symmetry-related reflections.

$\$ \mathrm{R}_{\text {work }}=\mathrm{S}|| \mathrm{Fobs}|-| \mathrm{Fcalc}||$ / $\mathrm{S}|\mathrm{Fobs}|$, where $|\mathrm{Fobs}|$ and $\mid$ Fcalc $\mid$ are the observed and calculated structure factor amplitudes respectively.

$\S \mathrm{R}_{\text {free }}$ is the same as $\mathrm{R}_{\text {work }}$ but calculated with a $5 \%$ subset of all reflections that was never used in crystallographic refinement. 
B Biochemical Journal Immediate Publication. Published on 03 Sep 2014 as manuscript BJ20140587

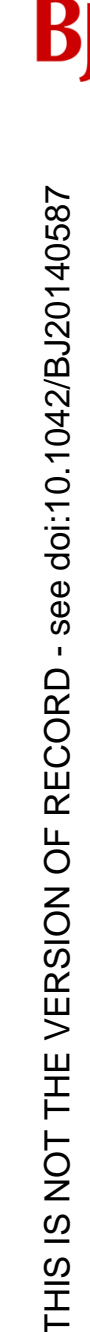

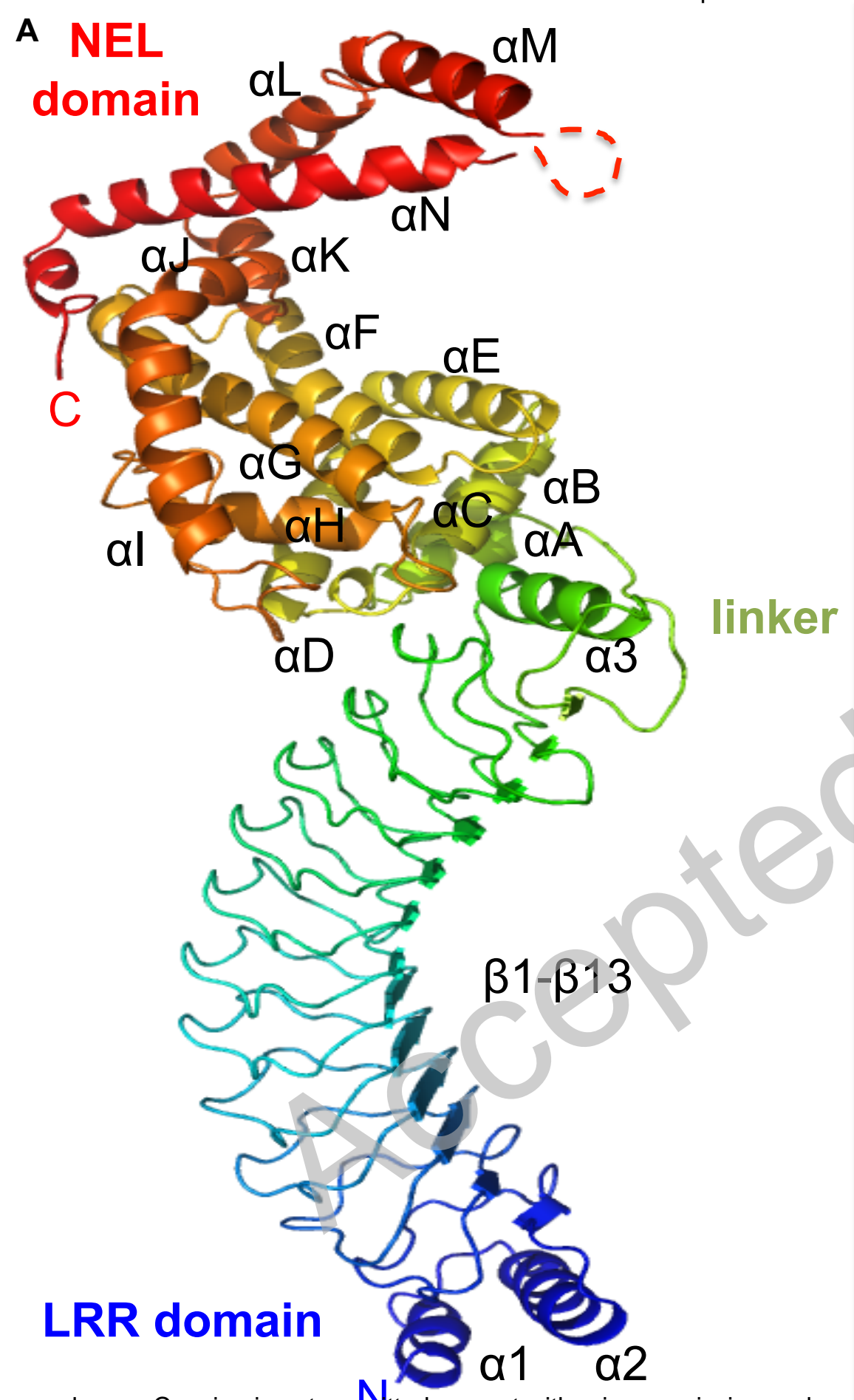

$L R R_{-} 1$

LRR 1

LRR_2

LRR_3

LRR 4

LRR 5

LRR 6

LRR 7

LRR 8

LRR 9

LRR 10

LRR_ 11

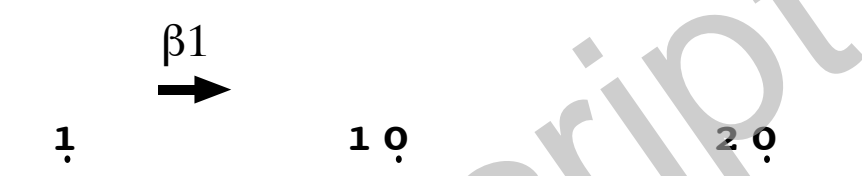

. K T E L RL K I L G L T T I RAYI P E...... Q I T T I I L D N N E I KSI P E N L Q G ........ N I K TL Y A N S N Q I TS I PATI P D T ...... . I Q E M EI S I NRI T E L P E RI P S ...... A L $Q$ S I DI F H N KI S C L P ENIL P E E ....... . L R Y L S V Y DNSI RTL PAHI P S E...... . I T H L N V Q S N S L T A L P E T I P P ....... G L K T L E A G E NAL T S L P A S L P P ....... E I OVILV S K N Q I T V L P E TI P P T ....... . I T TL DV S R NAL T NLPENI PAA ...... . L Q I M Q A S R N N L V R L P E S L P H F R G E G P Q P

LRR-12 $\stackrel{\beta 12}{\longrightarrow} \quad 10$ elebelelelel

LRR-12 TRI I VEYNPFSERTIQNMQRLMSSVDYQ

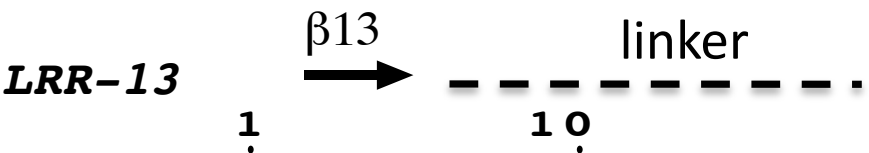

LRR-13 GPRVLFAMG DF S IVRVTRP 


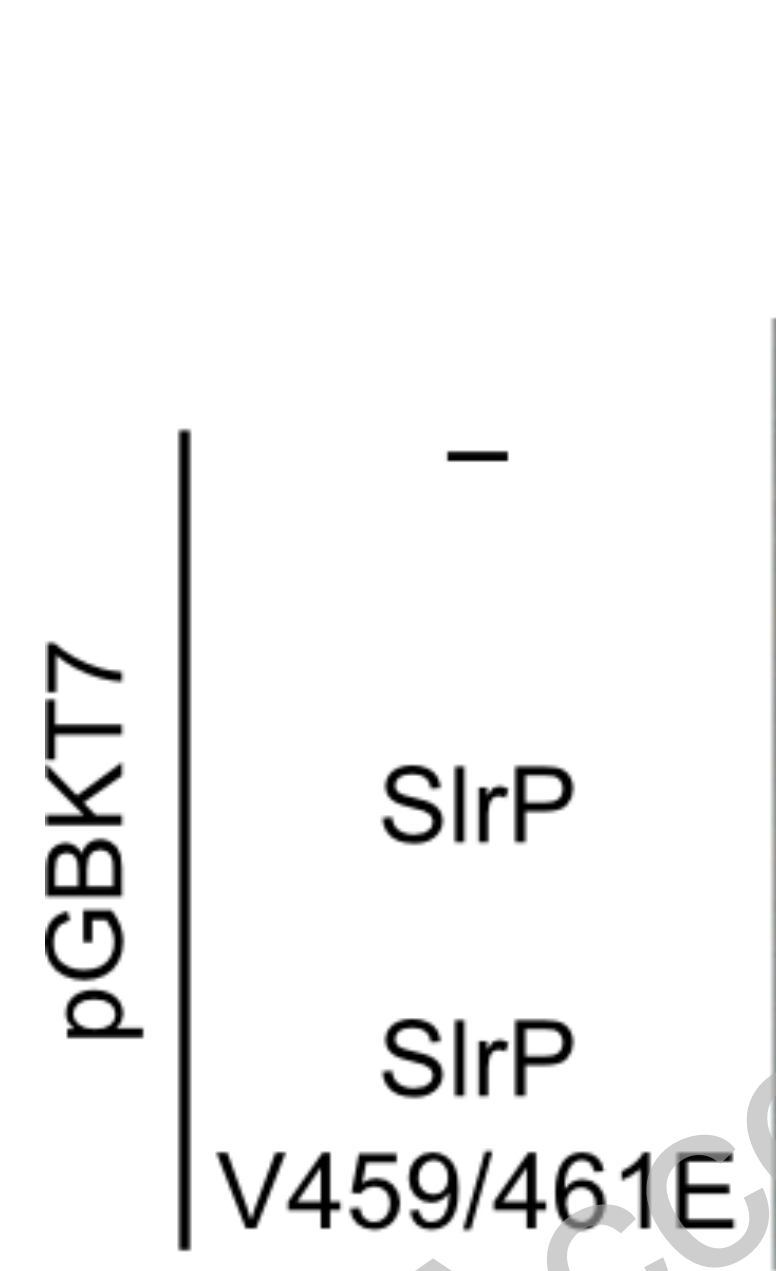

-Trp -Leu -His -Ade 
R Biochemical Journal Immediate Publication. Published on 03 Sep 2014 as manuscript BJ20140587
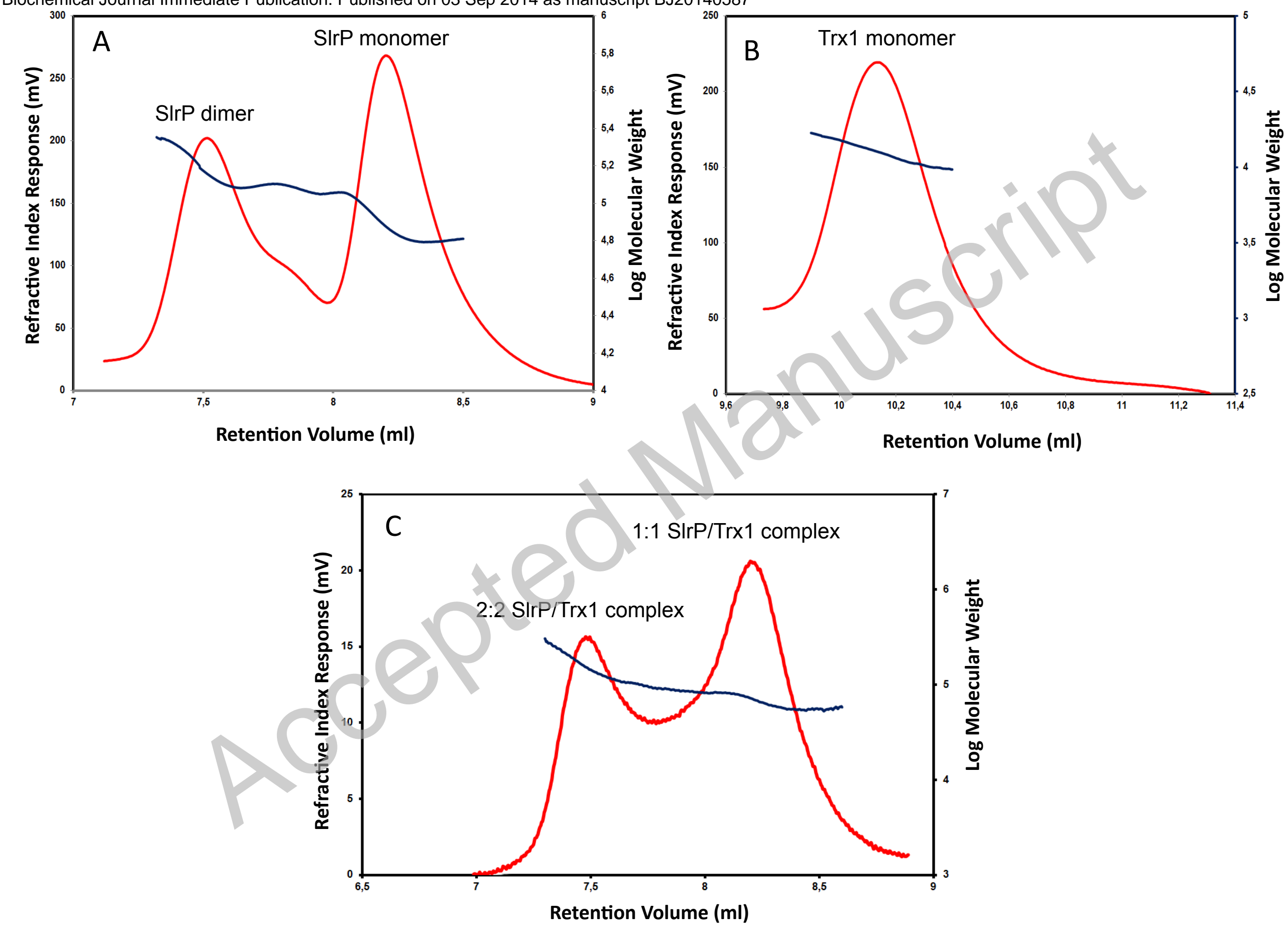

Licenced copy. Copying is not permitted, except with prior permission and as allowed by law.

(C) 2014 The Authors Journal compilation () 2014 Biochemical Society 


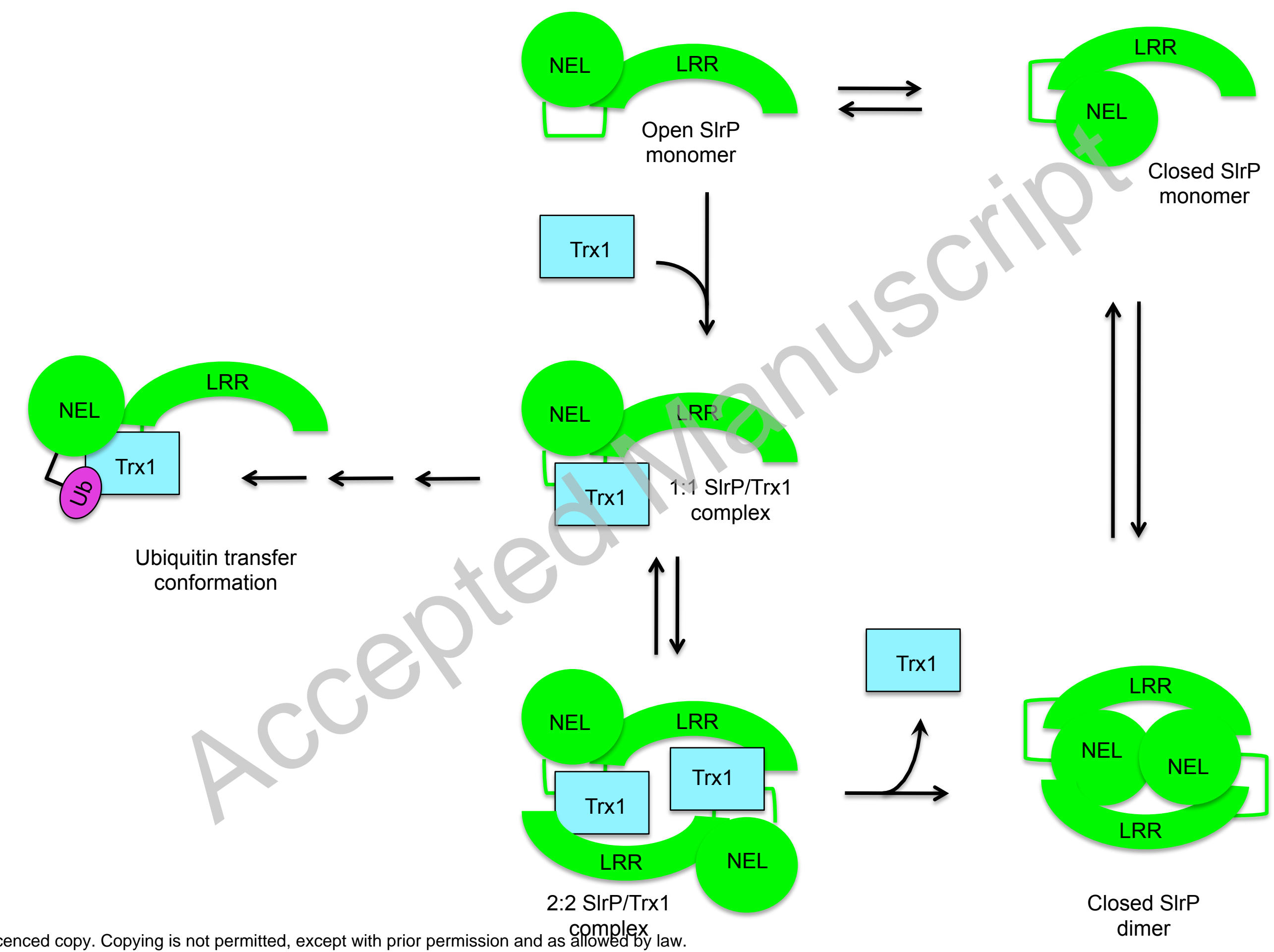

Licenced copy. Copying is not permitted, except with prior permission and as allowed by law. (C) 2014 The Authors Journal compilation ( 2014 Biochemical Society 